\title{
Very late restenosis of a sirolimus-eluting stent after left main coronary artery stenting
}

\author{
Stéphane Cook MD, Peter Wenaweser MD, Stephan Windecker MD
}

A 56-year-old woman was diagnosed with congestive heart failure (New York Heart Association functional class III) in the presence of slightly decreased left ventricular function as assessed by transthoracic echocardiography. The patient had been treated with high-dose chemotherapy (cyclophosphamide) for newly diagnosed systemic sclerosis with cutaneous and pulmonary involvement. She also had arterial hypertension, dyslipidemia and a positive family history of coronary artery disease. The patient denied having angina pectoris.

The patient underwent coronary angiography, which showed isolated, significant stenosis of the midshaft of the left main coronary artery (Figure 1A). The patient refused surgery and percutaneous coronary intervention was undertaken. The lesion was treated by direct stent implantation using a 3.0/13 mm sirolimus-eluting stent (Figure 1B) (Cypher, Cordis, USA) implanted with an inflation pressure of $1800 \mathrm{kPa}$, with a satisfactory result (Figure 1C). Six months later, a follow-up angiogram showed a good result without evidence of restenosis
(Figure 1D). Another angiogram performed because of chest pain 19 months after stent implantation confirmed a good long-term result (Figure 1E). Forty-three months after stent implantation, coronary angiography was repeated due to worsening symptoms of congestive heart failure and deterioration of left ventricular function. This time, the angiogram revealed a subtotal in-stent restenosis at the proximal stent edge (Figure 1F). The patient underwent coronary artery bypass surgery.

Drug-eluting stents have been shown to effectively reduce neointimal hyperplasia, and thus restenosis, compared with bare metal stents. Long-term follow-up of large-scale clinical trials indicate only a small rate of target lesion revascularization of approximately $1 \%$ per year beyond one year of stent implantation. The current example highlights, however, that late restenosis may occur with drug-eluting stents, requiring careful clinical follow-up, particularly with stents implanted into the left main coronary artery.

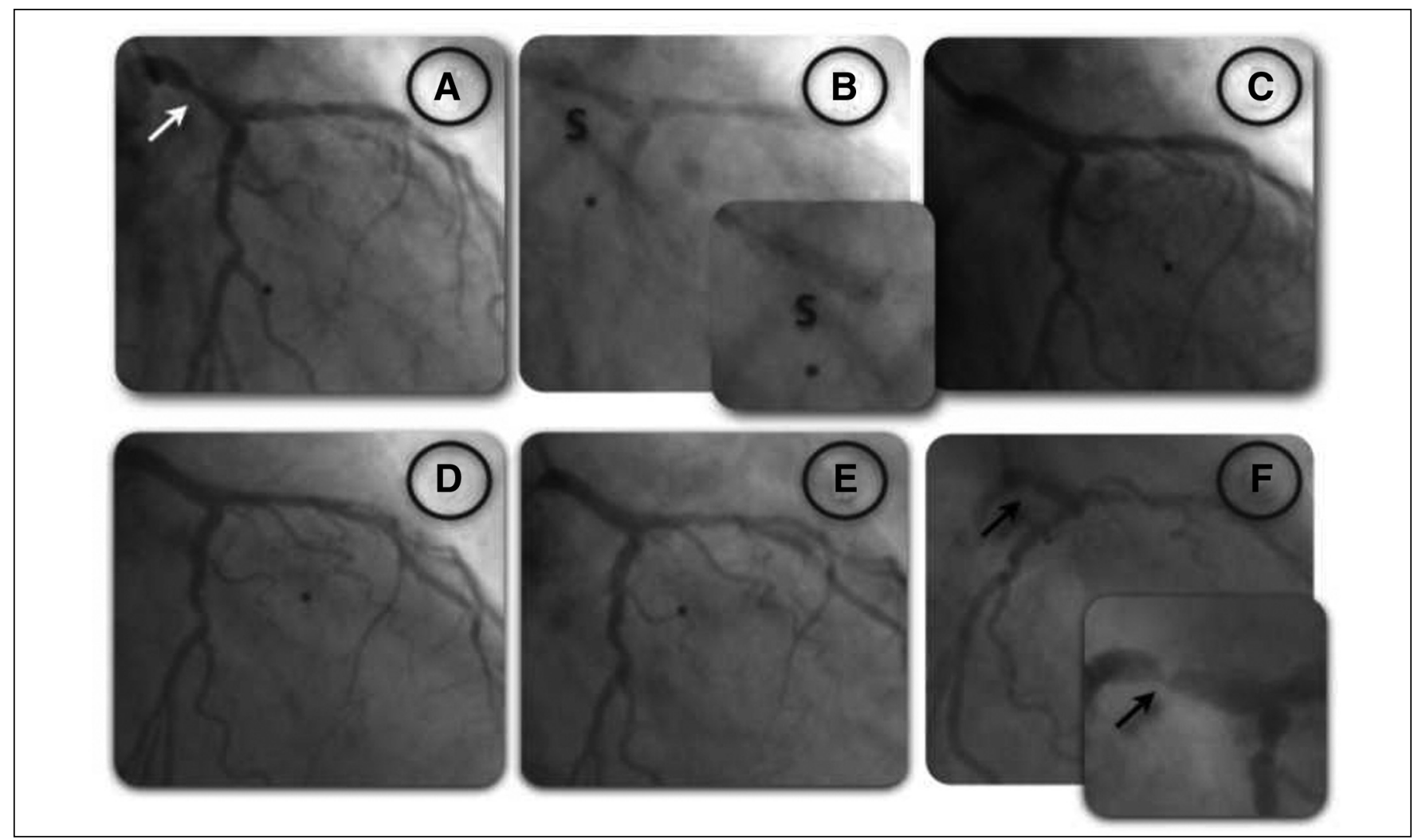

Figure 1) Coronary angiography showing signficant stenosis of the midshaft of the left main coronary artery (white arrow [A]); the treated lesion by direct stent implantation (S [B]) and its result (C) are shown. Follow-up coronary angiography at six months (D) and 19 months (E). F Angiography revealed a subtotal in-stent restenosis at the proximal stent edge (black arrow) 43 months after implantation

Department of Cardiology, University Hospital of Bern, Bern, Switzerland

Correspondence: Dr Stéphane Cook, Invasive Cardiology, University Hospital of Bern, 3010 Bern, Switzerland.

Telephone 41-31-632-21-11 ext 6049, fax 41-31-632-47-70, e-mail stephan.cook@insel.ch

Received for publication October 16, 2006. Accepted October 31, 2006 